\title{
Multi-component measuring device - completion, measurement uncertainty budget and signal crosstalk for combined load conditions
}

\author{
Sebastian Baumgarten, Dirk Röske and Rolf Kumme \\ Physikalisch-Technische Bundesanstalt (PTB), Bundesallee 100, 38116 Braunschweig, Germany
}

\begin{abstract}
This paper presents the completion and the measurement uncertainty budget of a multi-component measuring facility. The new facility is part of the $1 \mathrm{MN}$ force standard machine [1] at the Physikalisch-Technische Bundesanstalt (PTB). It enables the simultaneous generation of a torque in the range from $20 \mathrm{~N} \cdot \mathrm{m}$ to $2 \mathrm{kN} \cdot \mathrm{m}$ in addition to axial forces $20 \mathrm{kN}$ to $1 \mathrm{MN}$. This allows the characterization of measuring systems which require combined loads of axial forces $F_{z}$ and torques $M_{z}$, like friction coefficient sensors. The aim is a measurement uncertainty of $(\mathrm{k}=2)$ for $M_{z}<0.01 \%$ and $F_{z}<0.002 \%$. The physical model yields to extended measurement uncertainties $(k=2)$ for $20 \mathrm{~N} \cdot \mathrm{m}$ of $5.9 \cdot 10^{-5}$ and for the maximum load step $M_{z}=(2000 \pm 0.084) \mathrm{N} \cdot \mathrm{m}$.
\end{abstract}

\section{Section: RESEARCH PAPER}

Keywords: multi-component measurement; measurement uncertainty budget; torque; force; signal crosstalk; friction coefficient sensor

Citation: Sebastian Baumgarten, Dirk Röske and Rolf Kumme, Multi-component measuring device - completion, measurement uncertainty budget and signal crosstalk for combined load conditions, Acta IMEKO, vol. 6, no. 4, article 14, December 2017, identifier: IMEKO-ACTA-06 (2017)-04-14

Section Editor: Eric Benoit, University Savoie Mont Blanc, France

Received March 24, 2016; In final form January 19, 2017; Published December 2017

Copyright: (C) 2017 IMEKO. This is an open-access article distributed under the terms of the Creative Commons Attribution 3.0 License, which permits unrestricted use, distribution, and reproduction in any medium, provided the original author and source are credited

Corresponding author: Sebastian Baumgarten, e-mail: Sebastian.baumgarten@ptb.de

\section{INTRODUCTION}

There is an increasing number of measuring systems that can detect more than one force or torque component of these vectorial physical quantities. There is, therefore, an increasing need for traceability with regard to multi-component measurements. Realizations of such measuring facilities with sufficient measurement uncertainty and a suitable measuring range are complex and rare. PTB's hexapod [2] and the measuring facility at the Instituto Narionale di Ricerca Metrologica (INRiM) [3] are examples of such a realization. The PTB uses the infrastructure that is already available at such measuring facilities, to upgrade one facility by adding additional torque components. As a result of a project in PTB, within the $1 \mathrm{MN}$ force standard machine (1 MN FSM), torques can now be generated by means of a lever/band/mass system. This extension of the FSM allows the combination of a force measuring range from $20 \mathrm{kN}$ to $1 \mathrm{MN}$ with a torque measuring range from $20 \mathrm{~N} \cdot \mathrm{m}$ to $2 \mathrm{kN} \cdot \mathrm{m}$. This, in turn, extends the service range of the measuring facility, and measuring systems such as friction coefficient sensors or wheel load sensors can, thus, be investigated specifically. The measurement uncertainty budget (MUB) for $M_{\mathrm{z}}$ is presented.

\section{SET-UP}

The additional torque device has a modular set-up and can be mounted into or removed from the force flow of the 1-MN FSM. It works on the basis of the principle of a two-armed lever at the ends of which a force couple acts. The force couple is equal value which, although parallel to each other, act in the opposite direction to each other. The cross forces thus neutralize each other and all in all, an active torque $M_{\mathrm{z}}$ is realized. The forces are generated via two mass stacks that are located symmetrically on either side of the $1 \mathrm{MN}$ FSM (see Figure 1). Each of these mass stacks (see Figure 2) is composed of a lowerable set of masses. The mass disks are coupled to a metallic band. The metallic band is diverted by means of an airbearing rotor. The vertical gravitational force of the mass stacks becomes a horizontal tensile force. The metallic band is coupled to the lever arm and thus transmits the force onto the 


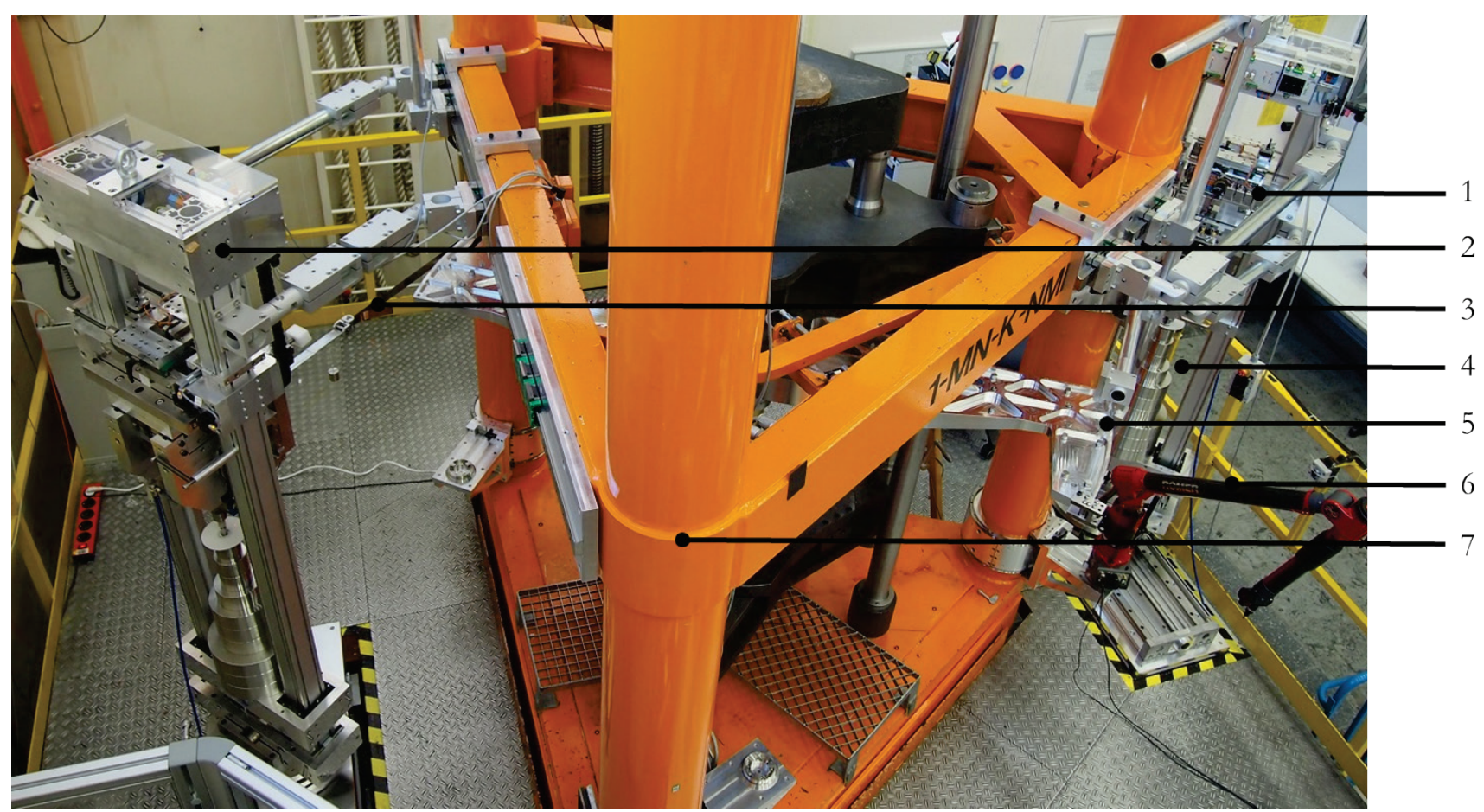

Figure 1. Multi-component measuring facility: 1 - mass stack A; 2 - mass stack B; 3 - metallic band for force application onto the lever; 4 - masses; 5 two-armed lever; 6 - coordinate measuring device, mounted onto a column support; 7 - 1 MN FSM.

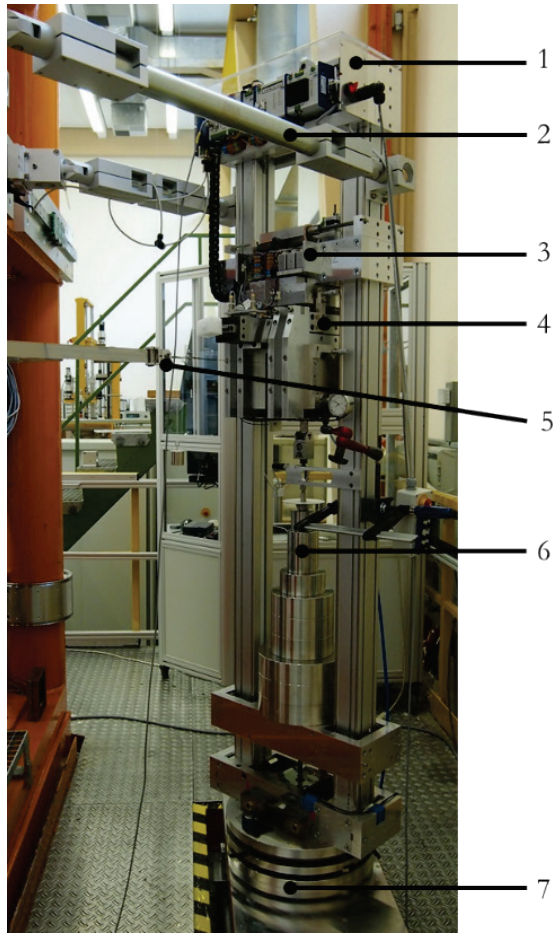

Figure 2. Mass stack B. Both mass stacks exhibit an identical design: 1 SPS control; 2 - Support elements resting against the frame of the $1 \mathrm{MN}$ FMS; 3 - block with step motors for the displacement and tilting of the airbearing head; 4 - air-bearing head with integrated rotor for force diversion; 5 - metallic band and coupling element for force application; 6 masses; 7 - rotational and linear table for position displacement of the mass stack.

system. Sensors and step motors stabilize the system position under load and changing load conditions. The synchronous triggering, monitoring and data acquisition are effected by EXCEL macros and a DMP 41.

\section{MEASUREMENT UNCERTAINTY BUDGET}

The following sections are only a summary of the important points of the measurement uncertainty budget, more details about this very comprehensive topic in [9]. A specific measurement uncertainty budget for the additional facility is presented. It includes a model, Figure 3, taking physical and geometric influence factors into account. This includes different factors, among other things, environmental influences, geometric characteristics, or the influence of the mass stacks. The influence of different influence factors on the measurement uncertainty and on the signal stability (e.g. friction inside the air bearing) have been investigated. In this case of application, also the realignment process of the mass stacks, the flatness errors of adaption parts and angular deviations must be taken into account. The model therefore encompasses a consideration of the system according to the vectorial components of $\boldsymbol{M}$ (1) and the analysis of the influence factors on the measurement uncertainty. In the coordinate system used,

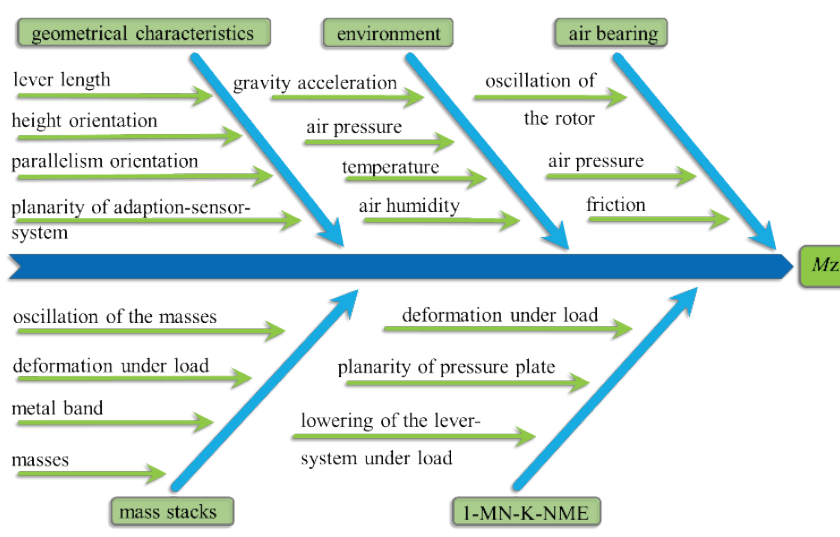

Figure 3. Overview of the acting influence factors in the form of an Ishikawa diagram for $M_{z}$ 
$M_{\mathrm{z}}$ is the torque, $l_{\mathrm{y}}$ is the lever length, and $F_{\mathrm{x}}$ is the applied force. The ideal case thus consists in the lever and the force vector lying in the $x$-y-plane and being oriented orthogonal to each other. An additional axial force $F_{z}$ can be applied onto the system by the $1 \mathrm{MN}$ FSM

$\vec{M}=\vec{F} \times \vec{l}=\left[\begin{array}{l}F_{x} \\ F_{y} \\ F_{z}\end{array}\right] \times\left[\begin{array}{l}l_{x} \\ l_{y} \\ l_{z}\end{array}\right]=\left[\begin{array}{l}F_{y} l_{z}-F_{z} l_{y} \\ F_{z} l_{x}-F_{x} l_{z} \\ F_{x} l_{y}-F_{y} l_{x}\end{array}\right]=\left[\begin{array}{l}M_{x} \\ M_{y} \\ M_{z}\end{array}\right]$.

Table 1 shows identified factors and their percentage weighting for the load steps $20 \mathrm{~N} \cdot \mathrm{m}$ and $2000 \mathrm{~N} \cdot \mathrm{m}$. Identical measurement uncertainty budgets have been established for each load step. According to the physical model, the measurement uncertainty $(\mathrm{k}=2)$ for the minimum load step $M_{\mathrm{z}}$ $=\left(20 \pm 1.2 \cdot 10^{-3}\right) \mathrm{N} \cdot \mathrm{m}$ and for the maximum load step $M_{\mathrm{z}}=$ $(2000 \pm 0.084) \mathrm{N} \cdot \mathrm{m}$.

\subsection{Local gravitational acceleration}

The local gravitational acceleration at the measuring station was determined by the Institute for Earth Measurement (IfE), Hannover, as being gloc $=9.812524 \mathrm{~ms}^{-2}$ with an expanded measurement uncertainty $(k=2)$ of $10 \mu \mathrm{ms}^{-2}$.

\subsection{Density and masses of the weight}

One set of weights includes the following load steps $1 \times 10 \mathrm{~N}, 2 \times 20 \mathrm{~N}, 1 \times 50 \mathrm{~N}, 3 \times 100 \mathrm{~N}$ and $3 \times 200 \mathrm{~N}$. The density of the material used for the cylindrical weights can be indicated as $\rho_{\mathrm{m}}=7979.7 \mathrm{~kg} \mathrm{~m}^{-3} \pm 2.0 \mathrm{~kg} \mathrm{~m}^{-3}(\mathrm{k}=2)$. The uncertainty components $(\mathrm{k}=2)$ lie for all masses $m_{\mathrm{m}}$ in a range $<5 \cdot 10^{-6} \mathrm{~kg}$ and are computed separately for each load step. The contribution to the measurement uncertainty budget never exceeds $1.33 \%$.

\subsection{Environment}

For the determination of the acting gravitational force, a buoyancy correction (2) was applied. The measuring facility is located in an air-conditioned hall. Changes in the ambient conditions are minimum. The actual values for the air pressure, the humidity and the temperature are acquired to compute the

Table 1. Measurement uncertainty budget for $20 \mathrm{~N} \cdot \mathrm{m}$ and $2000 \mathrm{~N} \cdot \mathrm{m}$.

\begin{tabular}{lcc}
\hline & \multicolumn{2}{c}{ Index of MUB } \\
Influence quantities & $\begin{array}{c}\left(\mathbf{2 0} \pm \mathbf{1 . 2} \cdot \mathbf{1 0}^{-3}\right) \\
\mathbf{N} \cdot \mathbf{m}\end{array}$ & $\mathbf{( 2 0 0 0 \pm \mathbf { 0 . 0 8 4 } ) \mathbf { ~ } \cdot \mathbf { m }}$ \\
\hline $\begin{array}{l}\text { Gravitational } \\
\text { acceleration }\end{array}$ & $0.05 \%$ & $0.12 \%$ \\
Ambient pressure & $<0.01 \%$ & $<0.01 \%$ \\
Air humidity & $<0.01 \%$ & $<0.01 \%$ \\
Temperature & $4.92 \%$ & $9.73 \%$ \\
Weight of the masses & $1.36 \%$ & $0.413 \%$ \\
Metallic bands & $<0.01 \%$ & $<0.01 \%$ \\
overlap & $45.24 \%$ & $89.53 \%$ \\
Lever length & $48.31 \%$ & $<0.01 \%$ \\
Air bearing friction & $0.06 \%$ & $0.11 \%$ \\
Metallic band & $<0.01 \%$ & $<0.01 \%$ \\
thickness & $0.04 \%$ & $0.07 \%$ \\
Height discrepancy & $<0.01 \%$ & $<0.01 \%$ \\
$\begin{array}{l}\text { Parallelism error } \\
\text { Angular error of the } \\
\text { pressure plate }\end{array}$ & & $<0.01 \%$ \\
$\begin{array}{l}\text { Angular error of the } \\
\text { adaption/sensor } \\
\text { system }\end{array}$ & $<0.01 \%$ & \\
\hline
\end{tabular}

MUB. Their influence on the MUB, however, lies in a range $<0.01 \%$. The ambient parameters from Table 1 for the MUB are the humidity $h_{\mathrm{L}}=42 \% \pm 5 \%$, the temperature $T_{\mathrm{L}}=21^{\circ} \mathrm{C} \pm 0.1^{\circ} \mathrm{C}$, and the ambient pressure $p_{\mathrm{L}}=1003.4 \mathrm{hPa} \pm 2 \mathrm{hPa}$

$F_{x}=m_{m} \cdot g_{l o c} \cdot\left(1-\frac{0,348 \cdot p_{L}-0,009 \cdot h_{L} \cdot e^{0,06 \cdot T_{L}}}{\left(273,15+T_{L}\right) \cdot \rho_{m}}\right)$.

\subsection{Lever length and thermal expansion}

A two-armed lever is used. A specified value of $999.92 \mathrm{~mm}$ applies to both sides. The length of the whole lever was calibrated at PTB's Coordinate Metrology Division; the result obtained was: $1999.882 \mathrm{~mm} \pm 0.028 \mathrm{~mm} \quad(\mathrm{k}=2)$. When calculating the total length, also the half of the thickness of the metallic bands for force application must be taken into account. The thickness is $0.08 \mathrm{~mm} \pm 0.001 \mathrm{~mm}$. The measurement uncertainty of the determination of the lever length represents the largest contribution to the MUB for $M_{\mathrm{z}}$. Due to the geometrical dimension of the lever, this uncertainty cannot be further reduced with the existing coordinate measuring machines.

The lever is made of an aluminium alloy. The thermal expansion for this alloy is $2 \cdot 10^{-5} \mathrm{~K}^{-1}$. Accordingly, temperature fluctuations of $0.1{ }^{\circ} \mathrm{C}$ have an influence of $4.92 \%$ on the MUB. The lever will later be replaced by another lever made of a temperature-stable INVAR alloy.

\subsection{Friction of the air bearings}

The air bearings do not provide absolutely friction-free force diversion. The influence of the friction inside the air bearing on the torque signal must therefore be investigated [5]. For this investigation, additional weights having a defined mass were applied. The weights are selected in such a way that, with the measuring chain used, a change in signal of practically one digit is expected. The measurements were repeated at all load steps up to $600 \mathrm{~N} \cdot \mathrm{m}$ and yielded the same result. A change of 1 digit, however, also corresponds to the signal stability of the measuring amplifier (DMP41 with low pass filter $0.04 \mathrm{~Hz}$ Butterworth), the influence has, thus, been estimated as being two digits. This corresponds to a maximum torque proportion of $3.1 \cdot 10^{-4} \mathrm{~N} \cdot \mathrm{m}$. The contribution to the MUB is constant across the load steps. The percentage contribution to the MUB for small load steps, $48.3 \%$, is therefore the largest.

\subsection{Influence of torsion under load}

Loading the system with a torque leads to a torsion of the adaption/sensor system. Torsion, in turn, leads to a reduction of the length of the metallic band between the lever and the unwinding point at the air bearing. The difference represents the overlapping of the metallic band on the side of the force generation $F_{\mathrm{x}}$ and, as an additional mass, it contributes accordingly to the torque $M_{\mathrm{z}}$. The proportion directly depends on the load step. The differential length is determined, by means of a laser sensor as being to $10 \mu \mathrm{m}$. The change in mass is determined by means of the band thickness $0.080 \mathrm{~mm} \pm 0.001 \mathrm{~mm}$, height $30.0 \mathrm{~mm} \pm 0.1 \mathrm{~mm}$ and density $7850 \mathrm{~kg} \mathrm{~m}^{-3} \pm 20 \mathrm{~kg} \mathrm{~m}^{-3}$ and it is taken into account for the torque calculation. The contribution to the MUB is $<0.01 \%$.

\section{GEOMETRICAL CHARACTERISTICS}

To calculate the MUB and the disturbing quantities, the orientation as well as the geometric deviation from the optimal orientation must be detected. Parallelism differences, angular 
deviations, tilts of the lever and height differences are part of these deviations.

A coordinate measuring device acquires the geometric characteristics. By scanning any given point, the coordinate measuring device, with the aid of various angular encoders, computes the spatial position in relation to the machine coordinate system. The quality of a measurement depends on the measurement process, on the user, on individual errors of the angular encoders as well as on the computation performed by the coordinate measuring device. We have assumed that the accumulation of the individual errors follows a Gaussian distribution. The hypothesis was checked - and confirmed - by repeated measurements and by means of a Shapiro-Wilk test [6] for the individual measurement processes.

Sine and cosine functions must be used to calculate the MUB according to (1). The problem is that the sensitivity coefficient often tends to be zero at small angles. For this reason, an upper estimation is used for the influence [7].

\subsection{Deviation in parallelism orientation}

For an ideal couple, both metallic bands must be exactly parallel to each other. Measurement points for the coordinate measuring device on the lever and on the air bearing serve as reference points to determine the angle. The uncertainty across the measurement process was estimated by averaging with $(k=2) 0.02^{\circ}$. Together with the fine adjustment of the angular orientation, a parallelism error of $0.072^{\circ}$ is obtained.

\subsection{Deviation in height orientation}

For the ideal orientation, the height of the force application point at the lever must be in agreement with the band unwinding point at the air bearing. Reference points are used for the height orientation of the system; with $45 \mu \mathrm{m}(\mathrm{k}=2)$ for the normal contribution throughout the measurement process. The stability of the height is given by differential height measurements with a laser sensor at the end of the lever and by the displacement of the air bearing via a step motor. The signal threshold level for the adjustment was laid down as being 100 $\mu \mathrm{m}$. If the signal threshold level and the contributions due to the uncertainties of differential height measurements and of the displacement by means of the step motor, then one obtains a total contribution of $186 \mu \mathrm{m}$. A reduction of the signal threshold level considerably reduces the uncertainty; however, the effort for the adjustment control then increases tremendously. A one-sided height difference of $186 \mu \mathrm{m}$, related to a band length of $1440 \mathrm{~mm}$, corresponds, at $1000 \mathrm{~N}$, to a negligible change in torque of $8.3 \cdot 10^{-6} \mathrm{~N} \cdot \mathrm{m}$. When loading with the $1 \mathrm{MN} \mathrm{FSM} \mathrm{with} F_{z}$, the adaptor/sensor/lever set-up lowers itself. This is due to elongations in the $1 \mathrm{MN}$ adjustment control and compression of the adapter/sensor system. For 500 $\mathrm{kN}$ this lowering amounts to about $2.6 \mathrm{~mm}$. This height difference to the air bearing is corrected automatically by the adjustment control when the load is changed. The orientation therefore remains stable, even under an $F_{z}$ load, in a range of $186 \mu \mathrm{m}$.

\subsection{Deviation in planarity of the pressure plate, adaptation and sensor}

The lever's tilt in relation to the ideal $x-y$-plane depends on the orientation of the adaptor/sensor system. The adaptor parts are mechanical components to mounting the sensors at the multi-component facility. Deviations lead to angular errors and, thus, to a tilt of the lever. The standard reference is the pressure plate of the $1 \mathrm{MN}$ FMS. Averaging over different measurement series provides an estimate of the flatness. This can be specified as $\alpha_{\mathrm{PP}}=0^{\circ} \pm 0.0178^{\circ}$. The angle refers to a tilt of the plane in relation to the ideal $\mathrm{x}$-y-plane. Correspondingly, an angular deviation $\alpha_{\mathrm{PP}}=0^{\circ} \pm 0.0178^{\circ}$ also applies to the lever.

In addition, the flatness errors accumulate due to the adaption parts, the sensor and their installation. The resulting angular error depends on the quality of the components and must therefore be determined separately for each adaptor/sensor system. In the case of the MUB described in Table 1 , an angular error $\alpha_{\mathrm{AP}}=0.18^{\circ} \pm 0.0201^{\circ}$ can be stated. The MUB does not take into account the orientation of the angular error. The error is upper estimated by considering it as being constant for all directions. According to the calibration results obtained by the Coordinate Metrology Division, the deflection of the lever due to its dead weight can be neglected.

\section{DISTURBING QUANTITIES}

The quantities considered as disturbing quantities are the shearing force $F_{\mathrm{y}}$, an additional axial force $F_{\mathrm{z}}$ and the bending moments $M_{\mathrm{x}}$ and $M_{\mathrm{y}}$. A nominal value 0 is the goal for all disturbing quantities. Deviations of the geometric orientation (essentially), however, result in an uncertainty for the nominal value; this applies to each quantity.

The computation is carried out separately for each torque load step and have to be recalculated for each adaptor/sensor system. For the system to which also Table 1 applies, at 2000 $\mathrm{N} \cdot \mathrm{m}, 0 \mathrm{~N} \cdot \mathrm{m} \pm 0.4 \mathrm{~N} \cdot \mathrm{m}$ is obtained for $M_{\mathrm{x}}$ and $3.49 \mathrm{~N} \cdot \mathrm{m} \pm$ $1.01 \mathrm{~N} \cdot \mathrm{m}$ for $M_{\mathrm{y}}$. The deviation from the nominal value for $M_{\mathrm{y}}$ is due to the acting force $F_{\mathrm{x}}$ and to an effective lever length $l_{\mathrm{z}}$ as a result of the lever's tilt. Due to adaption parts with smaller flatness errors, it is possible to reduce the lever's tilt as well as the resulting bending moments significantly. Table 2 shows the percentage contribution of the significant influence quantities on the uncertainty of $M_{\mathrm{y}}$ and $M_{\mathrm{x}}$. The influence quantities that are not mentioned there, see Table 1 , have a negligibly small influence on the MUB amounting to $<0.0001 \%$.

The disturbing quantities $F_{\mathrm{y}}$ and $F_{\mathrm{z}}$ were also computed from the geometric deviations. At the maximum load step 2000 $\mathrm{N} \cdot \mathrm{m}$, one obtains for the system $0 \mathrm{~N} \pm 2.6 \mathrm{~N}$ for $F_{\mathrm{y}}$ and $0 \mathrm{~N} \pm$ $0.3 \mathrm{~N}$ for $F_{z}$.

Disturbing quantities may cause the characteristic curve of a sensor to shift. The signal crosstalk as a function of these quantities is often difficult to describe [8]. With little technical effort, it is possible to use the measuring device asynchronously in order to, for example, estimate the sensitivity of a sensor to a certain disturbing quantity.

\section{SIGNAL CROSSTALK FOR $S_{\mathrm{MZ}}$}

The combined load conditions between $F_{\mathrm{z}}$ and $M_{\mathrm{z}}$ and the signal crosstalk of $S_{\mathrm{Fz}}$ and $S_{\mathrm{Mz}}$ were investigated based on the

Table 2. Significant influences on the MUB of $M_{\mathrm{x}}$ and $M_{\mathrm{y}}$ for $2000 \mathrm{~N} \cdot \mathrm{m}$.

\begin{tabular}{lcc}
\hline & \multicolumn{2}{c}{ Index of MUB } \\
Influence quantities & $\boldsymbol{M}_{\mathrm{x}} / \mathbf{( 0 . 4 \mathbf { ~ N } \cdot \mathbf { m } )}$ & $\boldsymbol{M}_{\mathrm{y}} / \mathbf{( 1 . 0 1 ~ \mathbf { N } \cdot \mathbf { m } )}$ \\
\hline $\begin{array}{l}\text { Height discrepancy } \\
\text { Parallelism error }\end{array}$ & $99.97 \%$ & $<0.01 \%$ \\
$\begin{array}{l}\text { Angular error of the } \\
\text { pressure plate }\end{array}$ & $0.02 \%$ & $<0.01 \%$ \\
$\begin{array}{l}\text { Angular error of the } \\
\text { adaption/sensor system }\end{array}$ & $<0.01 \%$ & $43.98 \%$ \\
\hline
\end{tabular}


example of a multi-component sensor (MCS) which is specially adapted to the auxiliary device. The subsequent objective being the traceability of industrial sensors, the measurement results will be used to derive and develop expedient and practical calibration procedures and sequences as well as evaluation and analytical procedures.

\subsection{Multi-component sensor}

The sensor has the nominal load ranges $F_{\mathrm{z}}=500 \mathrm{kN}$ and $M_{\mathrm{z}}=500 \mathrm{~N} \cdot \mathrm{m}$. Calibration for individual quantities was performed according to EN ISO 376 and DIN 51309. For $F_{z}$, the sensor achieved $<5 \cdot 10^{-4}$ for $>100 \mathrm{kN}$ and $<2 \cdot 10^{-3}$ for $<100 \mathrm{kN}$, and for $M_{\mathrm{z}}$, the clock- and anti-clockwise measurement uncertainty is $(k=2)<7 \cdot 10^{-4} . F_{\mathrm{z}}$ is kept constant for a measurement series, whereas $M_{\mathrm{z}}$ is varied; the next load step $F_{z}$ is then selected and $M_{\mathrm{z}}$ is varied again. This sequence must be observed to prevent the mass disks from coupling asymmetrically into the load frame of the $1 \mathrm{MN}$ force standard machine. Figure 4 shows the result of the combined loading for the torque signal $S_{\mathrm{Mz}}$. The represented signal is only the signal change caused by combined loading. To assess the influence of signal crosstalk quantitatively, Figure 5 shows the relative change of the signal based on the signal evolution from the calibration function of $M_{\mathrm{Z}}$. Without correction, the error share can reach $4 \%$. This error inherent in the system must therefore be taken into account. The signal crosstalk of $M_{z}$ on the bridges of $S_{\mathrm{Fz}}$ is negligibly small and is therefore not represented here.

\subsection{Analysis by means of multiple polynomial regression}

As shown in Figure 4, the signal behaviour can, as a matter of principle, be represented by means of a higher-dimension regression surface. The multiple polynomial regression (MPR) method was applied. Equation (3) describes the calculation of a parameter matrix $\Theta$ from the identity matrix $A$ and a signal matrix $Z$. Depending on the order you are aiming at for the solution, a minimum number of data points are required. For a

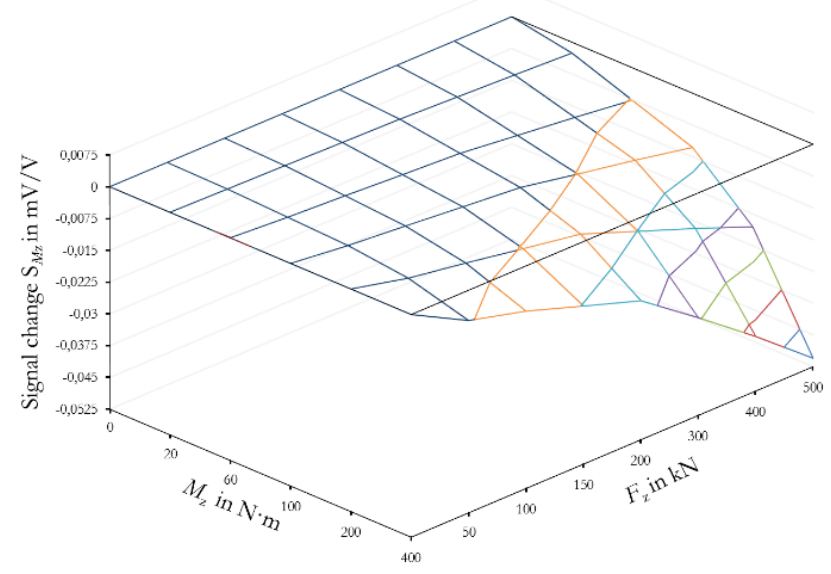

Figure 4. Torque signal change $S_{\mathrm{Mz}}$ by combined load conditions.

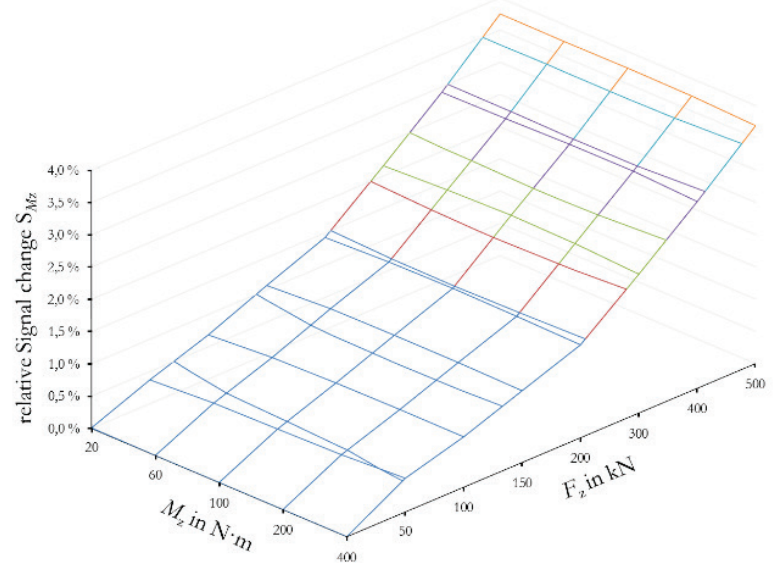

Figure 5. Relative signal change $S_{\mathrm{Mz}}$ by combined load conditions.

reliable statement with, e.g., a cubic approximate solution, five different $F_{\mathrm{z}}$ and $M_{\mathrm{z}}$ load steps - i.e. 25 independent data points - are necessary

$\theta=\left(A^{T} A\right)^{-1} A^{T} Z$.

Table 3 shows the set of parameters calculated and the coefficient of determination for the signal pattern from Figure 4. With (4) the cubic solution describes the signal pattern sufficiently well

$$
\begin{array}{r}
S_{M z}=a_{1} F_{z}+a_{2} M_{z}+a_{3} F_{z}{ }^{2}+a_{4} F_{z} M_{z}+a_{5} M_{z}{ }^{2}+\cdots \\
a_{6} F_{z}{ }^{3}+a_{7} F_{z}{ }^{2} M_{z}+a_{8} F_{z} M_{z}{ }^{2}+a_{9} M_{z}{ }^{3} .
\end{array}
$$

Especially for the range $>10 \%$ of the nominal load, the systematic influence can be reduced from $4 \%$ to $<0.5 \%$. The solution can only be applied to a limited extent to the lower load range. The MPR solution improves significantly with an increasing number of data points. This procedure is applied to both the loading and the unloading range. The inverse transformation of the signal values $S_{\mathrm{Mz}}$ and $S_{\mathrm{Fz}}$ to the input quantities $F_{z}$ and $M_{\mathrm{z}}$ is analogue. The next step is planned to consist of comparison measurements with various friction coefficient sensors in order to determine the specific signal crosstalk.

\section{CONCLUSIONS}

The extended relative measurement uncertainty $(\mathrm{k}=2)$ of the $1 \mathrm{MN}$ FSM is about $2 \cdot 10^{-5}$. The model provides an expanded relative measurement uncertainty for the additional measuring facility for torque generation of $M_{\mathrm{z}}=\left(20 \pm 1.2 \cdot 10^{-3}\right) \mathrm{N} \cdot \mathrm{m}$ up to $M_{\mathrm{z}}=(2000 \pm 0.084) \mathrm{N} \cdot \mathrm{m}$ for the maximum load. Comparison measurements with different torque reference transducers have shown very good repeatability; the reproducibility, however, is within a range of $<4.1 \cdot 10^{-4}$. The $<2 \cdot 10^{-4}$ goal has, thus, not been achieved yet. Most of the time, a measurement uncertainty $<1 \cdot 10^{-3}$ is sufficient for industrial sensors. Correspondingly, the measuring device is not yet listed in the catalogue of measuring facilities and PTB's

Table 3. Cubic solution parameter for the measurements of Figure 4.

Parameter for the cubic solution

\begin{tabular}{cccccccccc}
$\mathrm{a}_{1}$ & $\mathrm{a}_{2}$ & $\mathrm{a}_{3}$ & $\mathrm{a}_{4}$ & $\mathrm{a}_{5}$ & $\mathrm{a}_{6}$ & $\mathrm{a}_{7}$ & $\mathrm{a}_{8}$ & $\mathrm{a}_{9}$ & $\mathrm{R}^{2}$ \\
\hline $1 \cdot 10^{-4}$ & $-3.4 \cdot 10^{-3}$ & $-2.41 \cdot 10^{-7}$ & $-2.98 \cdot 10^{-7}$ & $-1.17 \cdot 10^{-7}$ & $2.83 \cdot 10^{-10}$ & $1.88 \cdot 10^{-11}$ & $9.2 \cdot 10^{-11}$ & $1.35 \cdot 10^{-10}$ & $7.89 \cdot 10^{-5}$ \\
\hline
\end{tabular}


Quality Management System. The characterization of the signal crosstalk by means of a specific multi-component sensor has shown that significant errors may occur when signal crosstalk is not taken into account. The multiple polynomial regression method allows the functional relation to be described precisely. Comparison measurements with industrial multi-component sensors from the screw industry will have to show whether these findings are applicable to other systems.

\section{REFERENCES}

[1] W. Weiler, M. Peters, H. Gassmann, H. Fricke, W.Ackerschott, "Die 1-MN-Normalmeßeinrichtung der PTB Braunschweig.", VDI-Z 120, 1978, pp. 1-6.

[2] D. Röske, "Metrological Characterization of a Hexapod for a Multi-Component Calibration Device", Proc. XVII IMEKO World Congress, Dubrovnik, Croatia, 2003, pp. 347-351.

[3] C. Ferrero, Li Qing Zhong, C.Marinari and E.Martino "New Automatic Multicomponent Calibration System with CrossedFlexure Levers", Proc. of ISMCR'93 - IMEKO TC-17, Torino, Italy, 1993.
[4] A. Robinson, „The Commissioning of the First UK National Standard Static Torque Calibration Machine“, 19th Conference on Force, Mass and Torque Measurement, Cairo, Egypt, 2005 pp.1-6.

[5] D. Peschel, D. Mauersberger, "Determination of the friction of aerostatic radial bearings for the lever-mass system of torque standard machines", Proc. of the XIII IMEKO World Congress, Turin (Italien), Sept. 5-9, 1994, vol. 1, pp. 216-220.

[6] S. S. Shapiro, M. B. Wilk "A analysis of variance test for normality”, Biometrika, Vol. 52, No. 3/4, 1965, pp. 591-611.

[7] D. Röske, "Uncertainty contribution in the case of cosine function with zero estimate - a proposal", IMEKO 2010 TC3, TC5 and TC22 Conferences, Pattaya, Chonburi, Thailand, November 22-25, 2010.

[8] A. Brüge, D. Röske, D. Mauersberger, K. Adolf "Influence of cross forces and bending moments on reference torque sensors for torque wrench calibration", XIX IMEKO World Congress, Lisbon (Portugal), Sept. 6-11, 2009.

[9] S.Baumgarten, H. Kahmann, D. Röske "Metrological characterization of a $2 \mathrm{kN} \cdot \mathrm{m}$ torque standard machine for superposition with axial forces up to $1 \mathrm{MN}$ ", Metrologia, Vol. 53, 2016, pp. 1165-1176. 